\title{
Apostando por la investigación en medicina de urgencias
}

\author{
Supporting research in emergency medicine
}

\author{
L. Daniel Sánchez-Arreola ${ }^{1,2,3 *}$
}

${ }^{1}$ Sociedad Mexicana de Medicina de Emergencia A.C.; ${ }^{2}$ Servicio de Emergencias, Centro Médico ABC Santa Fe; ${ }^{3}$ Subdirección Médica, Hospital General La Villa, Secretaría de Salud. Ciudad de México, México

A lo largo de los últimos 70 años la investigación científica en México ha evolucionado de manera satisfactoria, situación que ha propiciado no solo la creación de nuevos institutos nacionales de salud y de diversos centros de investigación orientados a la medicina y la biotecnología, sino también ha generado el desarrollo de políticas públicas relacionadas con el diagnóstico y la atención de enfermedades que durante muchos años han sido consideradas verdaderos problemas de salud pública ${ }^{1}$. Muchas de estas políticas permanecen vigentes y han permitido contrarrestar el potencial efecto a la salud pública de diversas enfermedades y padecimientos que de otra manera hubieran ocasionado una gran afectación a la población mexicana a lo largo de su historia.

Sin embargo, al igual que en la evolución de la medicina, el tiempo ha dejado al descubierto nuevos cambios en el estado de salud de la población y sus necesidades de atención médica tanto en lo individual como en lo colectivo. El advenimiento de las llamadas enfermedades crónicas no trasmisibles han denotado la necesidad de generar nuevas políticas y de realizar intervenciones y mecanismos que permitan la reorganización de los sistemas y la prestación de los servicios de salud, situación que exige a la par la generación de nuevos conocimientos científicos necesarios para entender las causas y los factores que influyen en el pronóstico y la supervivencia de la población actual, sobre todo en la fase aguda o en situación de emergencia².
De acuerdo con estadísticas de la Organización Panamericana de la Salud, se estima que 62 millones de personas en la región de las Américas viven con diabetes mellitus tipo 2, número que se ha triplicado desde el año de 1980 proyectando que el total de personas con diabetes mellitus alcanzará los 109 millones de personas en el 2040, incrementando drásticamente las posibilidades de mortalidad prematura, así como las complicaciones crónicas como enfermedades cardiovasculares, ceguera, nefropatía, problemas circulatorios, incrementando el riesgo de morir por enfermedades vasculares, trombosis y otras causas entre 2 y 3 veces más en comparación con personas sanas, complicaciones que hoy conforman las principales causas de mortalidad y representan la principal causa de ingreso en los servicios de urgencias $^{3}$.

Por otro lado, la aparición de padecimientos nuevos y poco conocidos, como la enfermedad causada por el COVID-19 del síndrome respiratorio agudo grave (SARS-CoV-2), que hoy ha sido la causa de más de 120 millones de personas infectadas y más de 2 millones 600 mil muertes en el mundo, ha constituido un reto y un verdadero motivo de reflexión para los investigadores, gerentes, administrativos y profesionales de la salud relacionados con las áreas críticas y los servicios de urgencias sobre la necesidad de retomar la investigación clínica como una verdadera prioridad enfocando su relevancia en la resolución de problemas y

\section{Correspondencia:}

*Luis Daniel Sánchez-Arreola

E-mail: dano23sa@ hotmail.com
Disponible en internet: 16-04-2021

Fecha de recepción: 19-03-2021

Fecha de aceptación: 22-03-2021

DOI: 10.24875/REIE.M21000010
Rev Educ Investig Emer. 2021;3(1):1-2

www.medicinadeemergencias.com

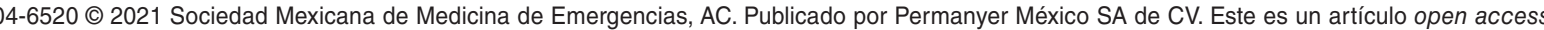
bajo la licencia CC BY-NC-ND (http://creativecommons.org/licenses/by-nc-nd/4.0/). 
la toma de decisiones sustentadas en una mayor producción de conocimiento científico ${ }^{4}$.

Es por eso por lo que en pleno siglo XXI podemos decir que estamos seguros de que no existen recetas o fórmulas mágicas que puedan ser usadas por los profesionales de la salud para enfrentar el reto de tratar estos padecimientos y los próximos, sino que es la investigación orientada a la medicina de urgencias y un flujo de información científica constante y accesible lo que asegurará en el futuro una práctica médica segura, acertada y basada en la evidencia.

Realizar acciones tangibles como promover la formación metodológica desde la etapa de formación clínica que ayuden a generar nuevas preguntas de investigación, crear nuevos órganos de difusión y divulgación científica serios, planificados y sustentados financieramente, promover la cultura del análisis de los resultados obtenidos en la práctica clínica diaria, generar nuevos indicadores, registros y reportes de casos, serán los pasos indispensables para avanzar hacia la medicina basada en la evidencia y la medicina basada en resultados de la práctica clínica del mundo real que hoy enfrentamos.

La investigación en medicina de urgencias debe ser hoy una prioridad no solo para cualquier sistema de salud, sino también para los profesionales que lo conforman, pues será solo la investigación el medio adecuado para impulsar el desarrollo profesional de los médicos y mejorar su preparación para ofrecer una medicina más segura para sus pacientes, situación que exigirá y se tendrán que enfrentar sin duda en la medicina del futuro.

Es por esto que al iniciar un nuevo bienio la Sociedad Mexicana de Medicina de Emergencia, conformada por una mesa directiva representada por ejemplares colaboradores y líderes en la medicina de urgencias, presenta este primer número por medio del cual refrendamos el compromiso de continuar trabajando arduamente para impulsar la generación de nuevos y novedosos programas educativos, así como de diversos contenidos científicos que fomenten la investigación y ayuden a la formación y desarrollo de las nuevas generaciones de médicos especialistas en medicina de urgencias, tomando en cuenta a la ciencia y el humanismo como su estandarte primordial, entendiendo que el conocimiento científico será siempre el mejor medio para mejorar la calidad de vida de nuestros pacientes desde un punto de vista personalista entendiendo su valor como seres humanos.

\section{Bibliografía}

1. Fajardo Dolci G. Ritmo y rumbo de la salud en México. Conversaciones con los secretarios de Salud 1982-2018. México: Fondo de Cultura Ecónomica; 2018.

2. Organización Panamericana de la Salud. Salud en las Américas+. Edición del 2017. Resumen: panorama regional y perfiles de país [Internet]. Washington D.C.: Organización Panamericana de la Salud, Organización Mundial de la Salud; 2017. Disponible en: https://iris.paho.org/handle/10665.2/34322

3. Organización Mundial de la Salud. Informe mundial sobre la diabetes [Internet]. Ginebra: Organización Mundial de la Salud; 2016. Disponible en: https://apps.who.int/iris/bitstream/handle/10665/254649/9789243565255-s pa.pdf;jsessionid=9719868B075228F12735FB62479F7BAB? sequence=

4. Dong $E$, Du H, Gardner L. An interactive web-based dashboard to track COVID-19 in real time. Lancet Inf Dis. 2020;20(5):533-4. 